\title{
Uso antenatal de corticosteróide e evolução clínica de recém-nascidos pré-termo
}

\author{
Antenatal corticosteroid use and clinical evolution of preterm newborn infants \\ Rede Brasileira de Pesquisas Neonatais*
}

\section{Resumo}

Objetivo: Descrever a freqüência de utilização de corticosteróide antenatal e a evolução clínica dos recém-nascidos pré-termo.

Métodos: Estudo observacional prospectivo tipo coorte de todos os neonatos com idade gestacional entre 23 e 34 semanas nascidos na Rede Brasileira de Pesquisas Neonatais entre agosto e dezembro de 2001. Os prontuários médicos foram revistos, as mães entrevistadas e os pré-termos acompanhados. A análise dos dados foi realizada com o teste do qui-quadrado, $t$ de Student, Mann-Whitney, ANOVA e regressão logística múltipla, com nível de significância de $5 \%$.

Resultados: Avaliaram-se 463 gestantes e seus 514 recémnascidos. As gestantes tratadas tiveram mais gestações prévias, consultas de pré-natal, hipertensão arterial e maior uso de tocolíticos. Suas crianças apresentaram melhores escores de Apgar no $1^{\circ}$ e $5^{\circ}$ minutos, menor necessidade de intervenção na sala de parto e menor SNAPPE II. Nasceram com maior peso e idade gestacional, receberam menos surfatante exógeno, ventilação mecânica e oxigenoterapia. Após regressão logística, o uso pré-natal de corticosteróides manteve de forma independente $o$ efeito protetor para as condições de nascimento e para a diminuição do tempo de ventilação mecânica e esteve associado com aumento na ocorrência de sepse neonatal.

Conclusão: $O$ uso do corticosteróide antenatal foi associado a melhor atendimento pré-natal. As crianças nasceram em melhores condições e tiveram melhor evolução, porém com maior risco de infecção.

J Pediatr (Rio J). 2004;80(4):277-84: Corticosteróide antenatal, prematuridade, ventilação mecânica, infecção, baixo peso.

\begin{abstract}
Objectives: To describe the use of antenatal corticosteroid and clinical evolution of preterm babies.

Methods: An observational prospective cohort study was carried out. All 463 pregnant women and their 514 newborn babies with gestational age ranging from 23 to 34 weeks, born at the Brazilian Neonatal Research Network units, were evaluated from August 1 to December 31,2001 . The data were obtained through maternal interview, analysis of medical records, and follow-up of the newborn infants. Data analysis was performed with the use of chi-square, $t$ Student, MannWhitney, and ANOVA tests and multiple logistic regression, with level of significance set at $5 \%$.
\end{abstract}

Results: Treatment was directly associated with the number of prenatal visits, with maternal hypertension and with the antenatal use of tocolytic agents. Babies from treated pregnant women presented better Apgar scores at the 1st and 5th minute, reduced need for intervention in the delivery room and lower SNAPPE II. They were born with higher birth weight, longer gestational age and needed less surfactant use, ventilation, and oxygenation time. After multiple logistic regression, the use of antenatal corticosteroid independently improved birth conditions, decreased ventilation time, being related to increased occurrence of neonatal sepsis.

Conclusions: The use of corticosteroid was associated with better prenatal care and birth conditions, better preterm evolution but higher risk of infection.

J Pediatr (Rio J). 2004;80(4):277-84: Antenatal glucocorticoids, prematurity, mechanical ventilation, infection, low birth weight.

* Participaram do estudo os seguintes pesquisadores, segundo local de coleta de dados:

Hospital das Clínicas, Faculdade de Medicina de Ribeirão Preto, Universidade de São Paulo (USP), Ribeirão Preto, SP: Francisco E. Martinez, Nelson J. Linhares e Maria L. S. Ferlin.

Centro de Atenção Integral à Saúde da Mulher, Universidade Estadual de Campinas (UNICAMP), Campinas, SP: Sérgio Marba e Abimael A. Netto. Hospital de Clínicas de Porto Alegre, Universidade Federal do Rio Grande do Sul (UFRGS), Porto Alegre, RS: Renato S. Procianoy e Natacha T. Uchoa. Instituto Fernandes Figueira, Fundação Oswaldo Cruz, Rio de Janeiro, RJ: José Maria A. Lopes e Olga Bomfim.

Hospital São Paulo, Escola Paulista de Medicina, Universidade Federal de São Paulo (UNIFESP), São Paulo, SP: Ruth Guinsburg, Maria Fernanda B. Almeida, Milton Miyoshi e Joice F. Meneguel.

Berçário Anexo à Maternidade do Hospital das Clínicas de São Paulo, Universidade de São Paulo (USP), São Paulo, SP: Cléa R. Leone, Lílian S. R. Sadeck e Flávio A. C. Vaz.

Hospital São Lucas, Pontifícia Universidade do Rio Grande do Sul (PUCRS), Porto Alegre, RS: Renato M. Fiori, Humberto H. Fiori e Manuel R. Pereira. Hospital das Clínicas, Faculdade de Medicina de Botucatu, Universidade do Estado de São Paulo (UNESP), Botucatu, SP: Cleide E. P. Trindade e Maria R. Betlin.

Fonte financiadora: esse estudo obteve apoio do Ministério da Saúde.

Artigo submetido em 10.03.04, aceito em 12.05.04. 


\section{Introdução}

Em 1972, Liggins \& Howie demonstraram que o uso antenatal de corticosteróide reduz significativamente a incidência de síndrome da dificuldade respiratória e mortalidade entre os pré-termos ${ }^{1}$. Numa meta-análise de 18 estudos controlados realizados entre 1972 e 1995, Crowley foi capaz de demonstrar que o uso antenatal de corticosteróide reduziu em $47 \%$ a incidência de síndrome da dificuldade respiratória e diminuiu em $40 \%$ a mortalidade entre as crianças nascidas pré-termo, além de reduzir em $52 \%$ a ocorrência de hemorragia intracraniana².

A despeito dos benefícios já demonstrados, o uso do corticosteróide antenatal, ainda hoje, parece não ser uma conduta terapêutica adequadamente disseminada. Em 13 estudos internacionais sobre uso de surfatante para prétermos publicados entre 1989 e 1994, ficou demonstrada claramente a irregularidade com que o corticosteróide foi utilizado. A taxa de utilização nesses artigos variou entre 0,8 e $81 \%^{3}$. Mais recentemente, em um levantamento de 11.440 partos ocorridos nos hospitais pertencentes à Canadian Neonatal Network, verificou-se seu uso em $59 \%$ dos partos pré-termo entre 24 e 34 semanas $^{4}$.

No nosso país, a figura parece não ser muito distinta. Como exemplo, pode-se citar o relato de administração de corticosteróide em cerca de $50 \%$ das pacientes que vieram a ter seu filho prematuramente em uma maternidade-escola 5 , ou pior, a irrisória taxa de $4 \%$ para os nascidos com menos de 34 semanas em sete maternidades públicas não-universitárias da cidade do Rio de Janeiro6.

O presente estudo visa descrever de forma prospectiva a freqüência de utilização do corticosteróide antenatal e a evolução clínica das crianças nascidas com 34 semanas ou menos de idade gestacional nos hospitais pertencentes à Rede Brasileira de Pesquisas Neonatais.

\section{Métodos}

Participaram deste estudo observacional prospectivo tipo coorte todos os neonatos com idade gestacional acima de 23 semanas e abaixo de 35 semanas nascidos nas maternidades e internados nas oito unidades neonatais pertencentes à Rede Brasileira de Pesquisas Neonatais no período de 1 de agosto a 31 de dezembro de 2001 e suas mães. Individualmente, cada instituição teve o protocolo de estudo aprovado pelo respectivo Comitê de Ética.

Foram excluídos os recém-nascidos com anomalias congênitas maiores ${ }^{7}$ ou síndromes cromossômicas. Também não foram considerados para estudo os neonatos cujas mães receberam corticosteróide antenatal para outra finalidade que não a indução da maturação fetal por exemplo, para o tratamento de doenças crônicas, como asma brônquica e colagenoses, entre outras.

Para cada nascimento de criança elegível a participar da pesquisa foi preenchida uma ficha de protocolo enquanto a mãe estava internada.
As variáveis maternas e neonatais foram obtidas por meio da revisão dos prontuários médicos, do cartão de prénatal e, quando possível, da pasta de pré-natal, além de entrevista com a mãe. Os dados foram coletados após a assinatura do termo de consentimento livre e esclarecido pela mãe.

Foram consideradas as seguintes características maternas: idade, gestações anteriores, filhos vivos, número de consultas de pré-natal, patologias durante a atual gestação, uso de tocolíticos e de corticosteróide antenatal, independente do número de doses, do tipo de corticosteróide (betametasona ou dexametasona) e do tempo de administração antes do parto.

Com relação aos recém-nascidos, registraram-se os seguintes dados: idade gestacional, adequação para a idade gestacional, Apgar no $1^{\circ}$ e $5^{\circ}$ minuto, condutas na sala de parto, SNAPPE II, evolução neonatal, tempo de internação e óbito.

A idade gestacional foi registrada em semanas, determinada por estimativa obstétrica, de acordo com a data da última menstruação materna, dados ultra-sonográficos ou exame neonatal através do método New Ballard 8 . A idade gestacional final foi considerada aquela calculada pela data da última menstruação ou pela ultra-sonografia fetal precoce quando a diferença entre esta data e a determinada pelo exame do recém-nascido era inferior a 1 semana. Quando a diferença era maior que 1 semana, a idade gestacional calculada pelo método de New Ballard era considerada.

A adequação do crescimento intra-uterino do recémnascido foi classificada por meio da curva de crescimento intra-uterino de Alexander et al. $^{9}$. Considerou-se como sendo adequado para a idade gestacional (AIG) o recémnascido com peso de nascimento entre os percentis 10 e 90, como pequeno para a idade gestacional (PIG) aquele abaixo do percentil 10 e como grande (GIG) o neonato que se encontrava acima do percentil 90.

Quando da necessidade de procedimentos de reanimação ao nascer, todas as unidades aplicam as normas da Academia Americana de Pediatria, adotadas pela Sociedade Brasileira de Pediatria. Desta forma, foi possível a quantificação do uso de ventilação com balão e máscara, intubação traqueal, massagem cardíaca e medicações ${ }^{10}$. Os escores de Apgar no $1^{\circ}$ e no $5^{\circ}$ minuto de vida foram registrados para todas as crianças $^{11}$.

O escore de gravidade da morbidade e do risco de mortalidade neonatal denominado Score for Neonatal Acute Physiology, Perinatal Extension, version II (SNAPPE-II), descrito por Richardson et al. ${ }^{12}$, foi aplicado a todas crianças na $12^{\mathrm{a}}$ hora de vida.

Quanto à evolução neonatal intra-hospitalar, foram compilados os seguintes desfechos clínicos e necessidade de tratamento:

- Síndrome do desconforto respiratório: diagnóstico clínico e radiológico.

- Síndrome de escape de ar: diagnóstico clínico e radiológico de pneumotórax, pneumomediastino e/ou enfisema intersticial. 
- Uso de surfatante exógeno.

- Necessidade de ventilação mecânica convencional, pressão positiva contínua em via aérea (continuous positive airway pressure - CPAP) ou outro modo de ventilação.

- Necessidade de oxigenoterapia.

- Doença pulmonar crônica: necessidade de oxigênio com 28 dias de vida e com 36 semanas de idade corrigida.

- Persistência do canal arterial (PCA), tratada com indometacina e/ou ligadura cirúrgica.

- Diagnóstico de sepse: definido como hemocultura positiva.

- Tratamento com antibioticoterapia por mais de 5 dias.

- Enterocolite necrosante: idade do diagnóstico clínicoradiológico ou cirúrgico, com classificação de I a III ${ }^{13}$ (todos os graus foram considerados).

- Hemorragia intraperiventricular (HIV), classificada segundo Papile et al. ${ }^{14}$. A classificação em graus I a IV foi feita por meio de ultra-sonografia transfontanelar realizada entre o quinto e o $14^{\circ}$ dia de vida ou após a ocorrência de alguma instabilidade clínica que pudesse causar ou agravar a hemorragia. Os graus III e IV foram anotados.

- Óbito intra-hospitalar: idade em horas ou dias calculada a partir do nascimento.

A análise dos dados foi realizada com o programa SPSS 10.0 para Windows. Foram utilizados os testes qui-quadrado ou exato de Fisher, $t$ de Student e Mann-Whitney, ANOVA e regressão logística múltipla. Para todos os testes, adotouse o nível de significância de $5 \%$.

A análise de regressão logística múltipla foi empregada para determinar as variáveis mais fortemente associadas às condições de nascimento, à necessidade de ventilação mecânica, à ocorrência de infecção e ao óbito. No caso das condições de nascimento, tomou-se o escore de Apgar de 10 minuto como variável dependente, classificado como $<3$ ou $\geq 3$. Na regressão logística referente à necessidade de ventilação mecânica, tomou-se como variável dependente o uso ou não de ventilador mecânico durante a internação. Para a avaliação da infecção, tomou-se como variável dependente a presença ou não de hemocultura positiva durante a internação. No caso da regressão referente ao óbito, teve-se como variável dependente o óbito ( $\operatorname{sim}$ ou não).

\section{Resultados}

Durante o período de estudo, 463 gestantes conceberam 514 recém-nascidos pré-termo com 34 semanas ou menos de idade gestacional. As principais características das gestantes estudadas, estratificadas segundo terem recebido ou não ao menos uma dose de corticosteróide antenatal, estão apresentadas na Tabela 1.

As gestantes que utilizaram os serviços dos hospitais da Rede eram jovens: 58 (12,5\%) delas tinham 18 anos ou menos. A maioria das pacientes era inexperiente, já que, para $68(14,7 \%)$, era a primeira gestação, e para 191, $(41,3 \%)$, o primeiro filho vivo; $109(23,6 \%)$ não fizeram uma única consulta no pré-natal.

Algumas características das gestantes que receberam corticosteróides diferiram significativamente das que não receberam. As gestantes tratadas tiveram mais gestações anteriores, mais consultas de pré-natal, diagnóstico mais freqüente de hipertensão e maior uso de tocolíticos.

Quanto às 514 crianças acompanhadas, algumas das características gerais do grupo como um todo e estratificadas segundo as mães terem recebido ou não corticosteróide antenatal estão apresentadas na Tabela 2.

Pode-se constatar que as crianças cujas mães receberam corticosteróide antenatal apresentavam algumas características diferentes das que não receberam. Ao nascer, essas crianças eram significativamente mais maduras, tanto em relação à idade gestacional como ao peso, além do predomínio do sexo feminino e do parto cesáreo quando comparadas às que não receberam tratamento antenatal com corticosteróides.

Tabela 1 - Principais características das gestantes estudadas, estratificadas segundo o uso ou não de corticosteróide antenatal

\begin{tabular}{lcccc}
\hline & $\begin{array}{c}\text { Todas } \\
\text { as mães }\end{array}$ & $\begin{array}{c}\text { Com } \\
\text { corticosteróide }\end{array}$ & $\begin{array}{c}\text { Sem } \\
\text { corticosteróide }\end{array}$ & p* \\
\hline Número & 463 & $284(61,3 \%)$ & $179(38,7 \%)$ & \\
Idade (anos) & $26,8 \pm 7,1$ & $27,4 \pm 7,3$ & $25,9 \pm 6,7$ & 0,06 \\
Gestações anteriores & $2,48 \pm 2,21$ & $2,66 \pm 2,31$ & $2,21 \pm 2,02$ & 0,03 \\
Filhos vivos & $1,25 \pm 1,62$ & $1,26 \pm 1,63$ & $1,22 \pm 1,62$ & 0,82 \\
Consultas de pré-natal & $3,53 \pm 3,1$ & $4,27 \pm 3,1$ & $2,41 \pm 2,5$ & $<0,01$ \\
Diabetes materno & $18(3,9 \%)$ & $14(3,0 \%)$ & $4(0,9 \%)$ & 0,14 \\
Infecção materna & $120(26 \%)$ & $77(16,6 \%)$ & $43(9,3 \%)$ & 0,54 \\
Bolsa rota > 18 horas & $93(20,1 \%)$ & $57(12,3 \%)$ & $36(7,7 \%)$ & 0,89 \\
Hipertensão & $146(31,6 \%)$ & $108(23,3 \%)$ & $38(8,2 \%)$ & $<0,01$ \\
Uso de tocolíticos & $87(18,8 \%)$ & $79(17,1 \%)$ & $8(1,7 \%)$ & $<0,01$ \\
\hline
\end{tabular}

* Comparação entre os grupos de gestantes com e sem corticosteróide antenatal. 
Tabela 2 - Algumas características das crianças avaliadas, estratificadas segundo as mães terem recebido ou não corticosteróide antenatal

\begin{tabular}{lcccc}
\hline & $\begin{array}{c}\text { Todas } \\
\text { as mães }\end{array}$ & $\begin{array}{c}\text { Com } \\
\text { corticosteróide }\end{array}$ & $\begin{array}{c}\text { Sem } \\
\text { corticosteróide }\end{array}$ & p* \\
\hline Número de crianças & 514 & $315(61,3 \%)$ & $199(38,7 \%)$ & \\
Peso (g) & $1.309 \pm 486$ & $1.353 \pm 443$ & $1.241 \pm 543$ & 0,01 \\
Idade gestacional (semanas) & $30,3 \pm 2,8$ & $30,8 \pm 2,2$ & $29,5 \pm 3,5$ & $<0,01$ \\
Pequenos para idade & $138(26,8 \%)$ & $86(27,3 \%)$ & $52(26,1 \%)$ & 0,77 \\
Sexo feminino & $247(48,1 \%)$ & $164(52,1 \%)$ & $83(41,7 \%)$ & 0,03 \\
Parto cesárea & $323(62,8 \%)$ & $214(67,9 \%)$ & $109(54,8 \%)$ & $<0,01$ \\
\hline
\end{tabular}

* Comparação entre mães com e sem corticosteróide.

A Tabela 3 apresenta as condições de nascimento, necessidade de cuidados na sala de parto e escore de gravidade SNAPPE II das crianças cujas mães receberam ou não corticosteróide antenatal.

As crianças das gestantes que receberam corticosteróide obtiveram escores de Apgar no $1^{0}$ e no $5^{\circ}$ minuto significativamente melhores, com conseqüente menor necessidade de intervenção na sala de parto. Com 12 horas de vida, a maior gravidade das crianças cujas mães não foram tratadas pôde ser constatada pelo maior valor encontrado do escore SNAPPE II.

Com a finalidade de verificar quais variáveis estariam associadas à condição de nascimento, tomou-se o escore de Apgar de 10 minuto como variável dependente, classificado como $<3$ ou $\geq 3$, e efetuou-se uma regressão logística. Primeiramente, avaliou-se quais variáveis estariam associadas individualmente ao escore de Apgar de $1^{\circ}$ minuto. As variáveis número de consultas de pré-natal, hipertensão materna, uso de corticosteróide, uso de tocolíticos, tipo de parto, idade gestacional, peso de nascimento e gênero estavam associadas de maneira estatisticamente significativa e, assim, entraram no modelo de regressão logística. Destas, permaneceram como estatisticamente significantes o uso antenatal de ao menos uma dose de corticosteróide, a idade gestacional e o fato de ser pequeno para a idade gestacional. As três variáveis apresentaram efeito protetor, ou seja, aumentaram a probabilidade do escore de Apgar ser maior que 3 no primeiro minuto. $O$ efeito protetor pode ser constatado na Tabela 4.

Essas crianças foram acompanhadas, e a Tabela 5 apresenta algumas informações sobre sua evolução clínica. Para efeito de análise, os recém-nascidos foram separados segundo a mãe ter recebido ou não ao menos uma dose de corticosteróide antenatal.

Tabela 3 - Condição de nascimento e necessidade de cuidados na sala de parto das crianças cujas mães receberam ou não corticosteróide antenatal

\begin{tabular}{lcccc}
\hline & $\begin{array}{c}\text { Todas } \\
\text { as mães } \\
(\mathbf{n}=\mathbf{5 1 4})\end{array}$ & $\begin{array}{c}\text { Com } \\
\text { corticosteróide } \\
\mathbf{( n = 3 1 5 )}\end{array}$ & $\begin{array}{c}\text { Sem } \\
\text { corticosteróide } \\
(\mathbf{n = 1 9 9 )}\end{array}$ & p* \\
\hline Apgar de 10 minuto $<3$ & $143(27,8 \%)$ & $61(19,4 \%)$ & $82(41,2 \%)$ & $<0,01$ \\
Apgar de 50 minuto $\geq 7$ & $429(83,5 \%)$ & $288(91,4 \%)$ & $141(70,9 \%)$ & $<0,01$ \\
Necessidade de: & & & & \\
$\quad$ Balão e máscara & $227(44,2 \%)$ & $136(43,2 \%)$ & $91(45,7 \%)$ & 0,57 \\
Intubação & $160(31,1 \%)$ & $71(22,5 \%)$ & $89(44,7 \%)$ & $<0,01$ \\
Massagem cardíaca & $37(7,2 \%)$ & $10(3,2 \%)$ & $27(13,6 \%)$ & $<0,01$ \\
$\quad$ Drogas & $29(5,6 \%)$ & $5(1,6 \%)$ & $24(12,1 \%)$ & $<0,01$ \\
SNAPPE II & $12,1 \pm 18,7$ & $10,5 \pm 14,9$ & $14,7 \pm 23,4$ & 0,01 \\
\hline
\end{tabular}

* Comparação entre mães com e sem corticosteróide. 
Tabela 4 - Regressão logística múltipla das variáveis associadas com os valores de Apgar de $1^{\circ}$ minuto (Apgar $<3=$ 0 ; Apgar $\geq 3=1$ )

\begin{tabular}{lccc}
\hline Variável & Odds ratio & IC* (95\%) & p \\
\hline $\begin{array}{l}\text { Uso antenatal } \\
\text { de corticosteróide }\end{array}$ & 2,47 & $1,43-4,30$ & $<0,01$ \\
$\begin{array}{l}\text { Idade gestacional } \\
\begin{array}{l}\text { Pequeno para } \\
\text { idade gestacional }\end{array}\end{array}$ & 1,38 & $1,19-1,60$ & $<0,01$ \\
\hline
\end{tabular}

* Intervalo de confiança.

O diagnóstico de membrana hialina foi comparável nos dois grupos, mas crianças cujas mães receberam corticosteróide antenatal precisaram ser tratadas menos vezes com surfatante exógeno e precisaram menos de ventilação mecânica e oxigenoterapia. Por outro lado, o tempo total de oxigenoterapia e a necessidade de oxigênio por tempo prolongado foram semelhantes entre os grupos.

A incidência de hemorragia intracraniana graus III e IV e a necessidade de tratamento medicamentoso ou cirúrgico do ducto arterioso patente não diferiu entre os dois grupos.

Em termos dos parâmetros relacionados à infecção, as crianças cujas mães receberam corticosteróide antenatal apresentaram maior incidência de hemoculturas positivas e maior uso de antibióticos, e uma maior porcentagem delas desenvolveu enterocolite necrosante de qualquer tipo, independentemente do número de ciclos de corticosteróides recebido. No entanto, a sobrevida foi maior entre essas crianças.
Para avaliar a influência das múltiplas variáveis associadas aos três principais desenlaces neonatais, ou seja, à necessidade de ventilação mecânica, à presença de hemocultura positiva e à sobrevida, realizou-se a regressão logística.

No caso da necessidade de ventilação mecânica, utilizou-se como variável dependente o fato da criança ter ou não recebido suporte de ventilação mecânica, e como variáveis independentes, aquelas que individualmente estiveram associadas com essa variável. Foram incluídas no modelo as variáveis número de consultas de pré-natal, uso antenatal de tocolíticos (sim ou não), uso antenatal de corticosteróide (sim ou não), idade gestacional, Apgar de 10 minuto e SNAPPE II. A Tabela 6 apresenta as variáveis que permaneceram no modelo após a regressão logística.

Pode-se constatar que o uso antenatal de corticosteróide, a idade gestacional ao nascimento e o escore de Apgar de $1^{\circ}$ minuto, após a regressão logística, mostraram-se como variáveis protetoras quanto à necessidade de ventilação mecânica. O SNAPPE II se apresentou como variável de risco. Todas as demais variáveis foram excluídas durante a regressão logística por não atingirem significância estatística.

Quanto à regressão logística das variáveis associadas à hemocultura positiva, utilizou-se como variável dependente o fato da criança ter tido ou não pelo menos um exame de hemocultura positivo durante sua internação. Como variáveis independentes, avaliaram-se aquelas que individualmente estiveram associadas com a hemocultura. Foram incluídas no modelo as seguintes variáveis: infecção materna (sim ou não), uso antenatal de corticosteróide (sim ou não), bolsa rota há mais de 18 horas (sim ou não), idade gestacional (em semanas), peso ao nascer (gramas), Apgar

Tabela 5 - Evolução clínica no período neonatal das crianças cujas mães receberam ou não corticosteróide antenatal

\begin{tabular}{|c|c|c|c|}
\hline & $\begin{array}{l}\text { Uso antenatal } \\
\text { de corticosteróide } \\
(n=315)\end{array}$ & $\begin{array}{c}\text { Sem } \\
\text { corticosteróide } \\
(n=199)\end{array}$ & $\mathbf{p}$ \\
\hline Membrana hialina & $147(46,7 \%)$ & $101(50,8 \%)$ & 0,37 \\
\hline Uso de surfatante & $85(27 \%)$ & $87(43,7 \%)$ & $<0,01$ \\
\hline Ventilação mecânica & $130(41,3 \%)$ & $114(57,3 \%)$ & $<0,01$ \\
\hline Dias em ventilação mecânica & $4,52 \pm 11,7$ & $6,57 \pm 15,9$ & 0,09 \\
\hline Escape de ar & $16(5,1 \%)$ & $15(7,5 \%)$ & 0,25 \\
\hline Necessidade de oxigênio & $218(69,2 \%)$ & $156(78,4 \%)$ & 0,02 \\
\hline Dias em oxigênio & $11,6 \pm 21,4$ & $14,1 \pm 29,3$ & 0,25 \\
\hline Oxigênio com 28 dias & $53(16,8 \%)$ & $35(17,6 \%)$ & 0,82 \\
\hline Oxigênio com 36 semanas & $33(10,5 \%)$ & $24(12,1 \%)$ & 0,57 \\
\hline Hemorragia intraperiventricular graus III ou IV & $23(7,3 \%)$ & $19(9,5 \%)$ & 0,41 \\
\hline Necessidade de tratamento do ductus arteriosus & $33(10,5 \%)$ & $28(14,1 \%)$ & 0,14 \\
\hline Hemocultura positiva & $60(19 \%)$ & $21(10,6 \%)$ & $<0,01$ \\
\hline Uso de antibióticos & $194(61,6 \%)$ & $96(48,2 \%)$ & $<0,01$ \\
\hline Enterocolite necrosante (todas) & $21(6,7 \%)$ & $5(2,5 \%)$ & 0,03 \\
\hline Enterocolite necrosante (grau de Bell $>1$ ) & $13(4,1 \%)$ & $3(1,5 \%)$ & 0,12 \\
\hline Sobrevida & $272(86,3 \%)$ & $135(67,8 \%)$ & $<0,01$ \\
\hline
\end{tabular}


Tabela 6 - Variáveis que permaneceram no modelo de regressão logística quanto à necessidade de ventilação mecânica dos recém-nascidos

\begin{tabular}{lccc}
\hline Variável & Odds ratio & IC* (95\%) & p \\
\hline $\begin{array}{l}\text { Uso antenatal } \\
\text { de corticosteróide }\end{array}$ & 0,51 & $0,29-0,89$ & 0,01 \\
Idade gestacional & 0,60 & $0,53-0,69$ & $<0,01$ \\
Apgar de 10 minuto & 0,85 & $0,77-0,93$ & $<0,01$ \\
SNAPPE II & 1,09 & $1,06-1,12$ & $<0,01$ \\
\hline
\end{tabular}

* Intervalo de confiança.

de $1^{\circ}$ minuto e SNAPPE II, síndrome da dificuldade respiratória (sim ou não), necessidade de ventilação mecânica (sim ou não), ocorrência de pneumotórax (sim ou não), persistência do canal arterial (sim ou não), presença de enterocolite necrosante ( $\operatorname{sim}$ ou não), hemorragia intraperiventricular graus III ou IV ( oxigênio após 36 semanas de idade corrigida (sim ou não). A Tabela 7 apresenta aquelas variáveis que permaneceram no modelo após a regressão.

Tabela 7 - Variáveis que permaneceram no modelo de regressão logística quanto à presença de hemocultura positiva nos recém-nascidos

\begin{tabular}{lccc}
\hline Variável & Odds ratio & IC* (95\%) & p \\
\hline $\begin{array}{l}\text { Uso antenatal } \\
\text { de corticosteróide }\end{array}$ & 2,87 & $1,52-5,39$ & $<0,01$ \\
Peso ao nascer & 0,99 & $0,99-1,00$ & 0,02 \\
Ventilação mecânica & 2,23 & $1,05-4,73$ & 0,04 \\
Oxigênio com 36 semanas & 2,67 & $1,32-5,42$ & 0,01 \\
\hline
\end{tabular}

* Intervalo de confiança.

Pode-se constatar que a única variável que permaneceu no modelo com efeito protetor foi o peso de nascimento. As variáveis uso antenatal de corticosteróide, ventilação mecânica e oxigênio com 36 semanas estiveram relacionadas com maior risco de hemocultura positiva.

$\mathrm{Na}$ regressão logística das variáveis associadas com óbito, utilizou-se como variáveis independentes aquelas que individualmente estiveram associadas de forma estatisticamente significante com a ocorrência do óbito. Foram incluídas no modelo as seguintes variáveis: número de consultas de pré-natal, uso antenatal de tocolíticos (sim ou não), uso antenatal de corticosteróide (sim ou não), hipertensão materna (sim ou não), idade gestacional (em semanas), Apgar de 10 minuto, SNAPPE II, necessidade de ventilação mecânica (sim ou não), ocorrência de pneumotórax (sim ou não) e hemorragia intraperiventricular graus III ou IV ( $\operatorname{sim}$ ou não). A Tabela 8 apresenta aquelas variáveis que permaneceram no modelo após a regressão.
O uso antenatal de tocolítico, a hipertensão materna e a idade gestacional foram variáveis que protegeram contra a ocorrência do óbito. O escore de gravidade SNAPPE II e a necessidade de ventilação mecânica foram fatores de risco de óbito.

Tabela 8 - Variáveis que permaneceram no modelo de regressão logística quanto à ocorrência de óbito dos recémnascidos

\begin{tabular}{lccc}
\hline Variável & Odds ratio & IC* (95\%) & p \\
\hline Uso antenatal de & & & \\
tocolítico & 0,23 & $0,08-0,61$ & $<0,01$ \\
Hipertensão materna & 0,43 & $0,20-0,86$ & 0,02 \\
Idade gestacional & 0,75 & $0,65-0,86$ & $<0,01$ \\
SNAPPE II & 1,02 & $1,01-1,04$ & 0,02 \\
Ventilação mecânica & 6,62 & $2,35-18,6$ & $<0,01$ \\
\hline
\end{tabular}

* Intervalo de confiança.

\section{Discussão}

Quando se pretende realizar um estudo cujo objetivo é avaliar a eficácia de alguma droga, o melhor modelo é o ensaio clínico duplo-cego controlado. No presente estudo, analisamos as repercussões do uso antenatal de corticosteróide por meio de um estudo de coorte observacional prospectivo, o que sem dúvida é uma limitação metodológica. No entanto, tendo em vista o conhecimento atual sobre o uso de corticosteróide antenatal ${ }^{2}$, seria antiético utilizar outro desenho do estudo.

O primeiro ponto que se destaca é que, de uma maneira geral, mais de $60 \%$ das mães avaliadas receberam corticosteróide antenatal. Se, por um lado, o conhecimento atual da eficácia dessa terapêutica indica que todas as mães em risco de parto prematuro deveriam ser tratadas ${ }^{2}$, por outro, isso nem sempre é possível ${ }^{15}$. Mesmo em países desenvolvidos, os índices de utilização do corticosteróide antenatal ainda estão muito aquém do desejável ${ }^{4}$. É interessante que as mães que receberam corticosteróide antenatal apresentaram características distintas das que não receberam. Podese dizer que as mães que receberam corticosteróide antenatal eram mais experientes e foram mais bem acompanhadas durante a gestação. No entanto, tanto as características das mães como a evolução das crianças não foram diferentes quando as mães receberam ciclos completos ou incompletos de corticosteróide antenatal. Desta forma, essas gestantes e suas crianças foram alocadas num único grupo de estudo.

O melhor acompanhamento pré-natal das gestantes que terminaram por receber o corticosteróide antenatal é um aspecto de grande importância: traz a indicação de que, quando existe maior oportunidade de contato com a paciente, melhoram as possibilidades de condutas preventivas. É interessante que o fato de algumas mães serem hipertensas não impediu que recebessem a droga. Desde que o uso de corticosteróide antenatal foi sugerido como uma maneira de 
amadurecer a função pulmonar dos recém-nascidos prétermo, sugeriu-se que a hipertensão materna poderia vir a ser uma contra-indicação à terapêutica ${ }^{1}$. Ainda hoje, este é um motivo de discussão ${ }^{15}$, mas não se constituiu uma limitação entre as mães avaliadas. Ao contrário, a hipertensão foi associada com maior número de tratamentos. Isso nos sugere que, mais importante que o temor do uso do corticosteróide na hipertensão foi o acompanhamento mais estreito que essas pacientes receberam, aumentando a possibilidade da oferta do corticosteróide. A associação entre o uso de tocolíticos e do corticosteróide vem a favor da hipótese de que essas mães tiveram um melhor atendimento de uma maneira geral. No caso de trabalho de parto prematuro, pode-se lançar mão do uso de tocolíticos com a finalidade de reverter o processo ou ganhar tempo para que o corticosteróide possa desempenhar sua função ${ }^{2}$.

Assim como nas mães, as características das crianças cujas mães receberam o corticosteróide antenatal eram diferentes das que não foram tratadas. Eram crianças mais velhas e mais pesadas e que nasceram preferencialmente por parto cesárea. O maior tempo de permanência intraútero dessas crianças, assim como a maior incidência de parto cesáreo, possivelmente são reflexos diretos do meIhor acompanhamento pré-natal dessas mães, e não efeito direto da droga.

A análise das condições de nascimento das crianças trouxe informações muito interessantes. De uma maneira geral, as crianças nascidas com menos de 34 semanas de gestação constituíram um grupo de alto risco. Quase metade delas necessitou de suporte com balão e máscara na sala de parto e cerca de 1/3 requereu intubação traqueal. Em outras palavras, essas crianças devem nascer em local preparado para suporte adequado na sala de parto. Nos serviços avaliados, esse suporte parece ter sido muito eficaz, já que, com 5 minutos, mais de $80 \%$ das crianças estavam em excelentes condições de vitalidade.

O grupo de neonatos expostos ao uso antenatal de corticosteróide apresentou melhores condições de nascimento. Quando se avaliou a necessidade de intervenção na sala de parto, ficou claro que as crianças cujas mães receberam corticosteróide nasceram em melhores condições. Elas necessitaram de menos condutas agressivas na sala de parto, tais como intubação traqueal, massagem cardíaca ou uso de adrenalina. Deve-se ressaltar que o efeito protetor do corticosteróide antenatal ainda pôde ser detectado com 12 horas de vida por meio do escore de gravidade SNAPPE II. O efeito protetor do corticosteróide, assim como o decorrente da idade gestacional e do retardo de crescimento intra-uterino sobre as condições de nascimento, permaneceram após a regressão logística. O fato de favorecer melhores condições de nascimento é um importante benefício da terapia antenatal com corticosteróide que não tem sido mas deve ser mais amplamente divulgado ${ }^{16}$.

A primeira ação benéfica descrita no uso antenatal de corticosteróide foi o amadurecimento pulmonar ${ }^{1}$. Pode-se dizer, pela análise individual das variáveis feita no presente estudo, que as crianças cujas mães receberam corticosteróide antenatal apresentaram melhor desempenho respira- tório. Ao contrário do apregoado na literatura ${ }^{17}$, o diagnóstico de membrana hialina foi comparável nos dois grupos. No entanto, as crianças cujas mães receberam corticosteróide antenatal precisaram de menos surfatante exógeno, menos suporte por ventilação mecânica e oxigênio e permaneceram por menos dias em ventilação artificial mecânica. Por outro lado, o tempo total de oxigenoterapia e a necessidade de oxigênio por tempo prolongado foram semelhantes nos dois grupos. Após a regressão logística, o uso antenatal de corticosteróide, a idade gestacional e o escore de Apgar de 10 minuto se mostraram como variáveis protetoras quanto à necessidade de ventilação mecânica. Por outro lado, o SNAPPE II se apresentou como fator de risco, ou seja, quanto maior seu valor, maior a necessidade de ventilação. Evidentemente, isso era de se esperar, já que se trata de um escore de gravidade ${ }^{12}$.

O uso antenatal de corticosteróide é descrito como fator protetor quanto à presença de hemorragia intraperiventricular $^{2}$ e, possivelmente, fator de risco para infecção neonatal $^{18}$. Nos pacientes avaliados no presente estudo, o efeito protetor quanto à hemorragia não pôde ser demonstrado.

As crianças cujas mães foram tratadas com corticosteróide antenatal apresentaram maior incidência de hemoculturas positivas e maior uso de antibióticos, e uma maior porcentagem delas desenvolveu enterocolite necrosante de qualquer tipo. Como as características iniciais das crianças eram muito diferentes, foi feita a regressão logística visando depurar quais fatores estariam realmente associados ao risco de infecção, sumarizado como presença de hemocultura positiva. Ao final da análise, permaneceram o uso antenatal de corticosteróide, o peso ao nascer, a ventilação mecânica e a necessidade de oxigênio com 36 semanas como significantes. Destas, somente a variável peso ao nascer se apresentou como fator protetor, e as demais, como era de se esperar, apresentaram-se como fatores de risco. Deve-se ressaltar que o uso de ventilação mecânica e de oxigênio com 36 semanas é associado à infecção tardia, remetendo a fatores relacionados aos cuidados na unidade. A evidência apresentada pela literatura a respeito do tratamento antenatal com corticosteróide e risco de infecção é conflitante. Enquanto a meta-análise de Crowley ${ }^{2}$ não aponta para maior risco, outros autores sugerem que, como demonstrado aqui, o risco de infecção está aumentado 4,19 . Como a literatura aponta que os efeitos benéficos da terapêutica são menores após 34 semanas de gestação, tem-se sugerido que a aplicação da terapêutica para as maiores de 34 semanas se limite a crianças com imaturidade pulmonar comprovada20.

Sem dúvida, a sobrevida é o desenlace mais importante da presente análise. A sobrevida é um parâmetro que, de certa forma, sumariza o sucesso de todo o conjunto de ações aplicadas ao paciente de risco. As crianças cujas mães receberam corticosteróide antenatal sobreviveram mais. Para a análise dos fatores associados à sobrevida, a realização da regressão logística é fundamental.

Muito interessante constatar que os fatores perinatais se mantiveram fortemente associados com a sobrevida. 0 uso antenatal de tocolíticos, a presença de hipertensão 
materna e a maior idade gestacional se apresentaram como fatores que aumentaram a possibilidade de sobrevivência - todos fatores sobre os quais o pediatra tem pouca possibilidade de intervir. Nota-se que o uso antenatal de corticosteróide não foi considerado significante na análise. De qualquer forma, permanece a forte indicação de que os cuidados pré-natais são fundamentais no prognóstico da criança.

Por outro lado, o escore SNAPPE II e a ventilação mecânica foram associados a maior risco de óbito. O SNAPPE II registra as condições da criança nas primeiras 12 horas de $v_{i d a}{ }^{12}$ e, como no caso dos outros parâmetros, indica a forte influência das condições perinatais no prognóstico da criança.

A necessidade de ventilação mecânica teve enorme peso no desenlace final da criança. Apesar dos grandes avanços que se tem verificado no desenvolvimento de novos respiradores e técnicas de ventilação mecânica, nossos dados indicam que a necessidade de ventilação mecânica é um forte risco para a sobrevida da criança. Por outro lado, deve-se atentar para o fato de que são as crianças mais graves que necessitam de suporte ventilatório.

Concluindo, este estudo apresenta uma coorte de crianças atendidas nos hospitais universitários pertencentes à Rede Brasileira de Pesquisas Neonatais e pode servir de base de comparação para os demais serviços brasileiros. As mães que receberam corticosteróide antenatal apresentavam características que indicam melhor atendimento pré-natal, sendo que as suas crianças nasceram maiores e com maior idade gestacional. O uso antenatal de corticosteróide foi diretamente associado a melhores condições de nascimento e a maior risco de infecção.

\section{Referências}

1. Liggins GC, Howie RN. A controlled trial of antepartum glucocorticoid treatment for prevention of the respiratory distress syndrome in premature infants. Pediatrics. 1972;50:515-25.

2. Crowley P. Prophylactic corticosteroids for preterm birth. Cochrane Database Syst Rev. 2002;(3):CD000065.

3. Ryan CA, Finer NN. Antenatal corticosteroid therapy to prevent respiratory distress syndrome. J Pediatr. 1995;126:317-9.

4. Chien L, Ohlsson A, Seshia MMK, Boulton J, Sankaran K, Lee SK. Variations in antenatal corticosteroid therapy: a persistent problem despite 30 years of evidence. Obstet Gynecol. 2002;99:401-8.

5. Albuquerque ICC, Amorim MMR, Meneses J, Katz L, Santos LC. Avaliação do impacto da corticiterapia antenatal para a aceleração da maturidade pulmonar fetal nos recém-nascidos em maternidade escola brasileira. RBGO. 2002;24:655-61.
6. Krauss-Silva L, Costa TP, Reis AF, Iamada NO, Azevedo AP, Albuquerque CP. Avaliação da qualidade da assistência hospitalar obstétrica: uso de corticóides no trabalho de parto prematuro. Cad Saúde Pública. 1999;15:817-29.

7. Chung CS, Myrianthopoulos NC. Congenital anomalies: mortality and morbidity, burden and classification. Am J Med Genet. 1987;27:505-23.

8. Ballard JL, Khoury JC, Wedig K, Wang L, Eilers-Walsman BL, Lipp R. New Ballard Score, expanded to include extremely premature infants. J Pediatr. 1991;119:417-23.

9. Alexander GR, Himes JH, Kaufman RB, Mor J, Kogan M. A United States National Reference for fetal growth. Obstet Gynecol. 1996;87:163-8.

10. Niermeyer $S$, Kattwinkel J, Van Reempts $P$, Nadkarni V, Phillips $B$, Zideman D, et al. International Guidelines for Neonatal Resuscitation: An excerpt from the Guidelines 2000 for Cardiopulmonary Resuscitation and Emergency Cardiovascular Care: International Consensus on Science. Contributors and Reviewers for the Neonatal Resuscitation Guidelines. Pediatrics. 2000;106(3): E29.

11. Apgar V. A proposal for a new method of evaluation of the newborn infant. Curr Res Anesth Analg. 1953;32:260-7.

12. Richardson DK, Corcoran JD, Escobar GJ, Lee SK. SNAP-II and SNAPPE-II: Simplified newborn illness severity and mortality risk scores J Pediatr. 2001;138:92-100.

13. Walsh MC, Kliegman RM. Necrotizing enterocolitis: treatment based on staging criteria. Pediatr Clin North Am. 1986;33: 179-201.

14. Papile LA, Burstein J, Burstein R, Koffler $H$. Incidence and evolution of subependymal and intraventricular hemorrhage: a study of infants with birth weights less than $1,500 \mathrm{gm}$. J Pediatr. 1978;92:529-34

15. Bloom SL, Leveno KJ. Corticosteroid use in special circumstances: preterm ruptured membranes, hypertension, fetal growth restriction, multiple fetuses. Clin Obstet Gynecol. 2003;45: 150-60.

16. Gardner MO, Goldenberg RL, Gaudier FL, Dubard MB, Nelson KG, Hauth JC. Predicting low Apgar scores of infants weighing less than 1000 grams: the effect of corticosteroids. Obstet Gynecol. 1995;85:170-4.

17. Banks BA, Macones G, Cnaan A, Merrill JD, Ballard JD, Ballard RA, et al. Multiple courses of antenatal corticosteroids are associated with early severe lung disease in preterm neonates. J Perinatol. 2002;22:101-7.

18. Vermillion ST, Soper DE, Chasedunn-Roak J. Neonatal sepsis after betamethasone administration to patients with preterm premature rupture of membranes. Am J Obstet Gynecol. 1999; 181:320-7.

19. Gunkel JH, Mitchell BR. Observational evidence for the efficacy of antenatal steroids from randomized studies of surfactant replacement. Am J Obstet Gynecol. 1995;173:281-5.

20. ACOG Committee Opinion. Antenatal corticosteroid therapy for fetal maturation. Obstet Gynecol. 2002;99:871-3.

Correspondência:

Francisco Eulógio Martinez

Departamento de Puericultura e Pediatria

Faculdade de Medicina de Ribeirão Preto - USP

Av. Bandeirantes, 3900

CEP 14049-900 - Ribeirão Preto, SP

Fone: (16) 602.2808 - Fax: (16) 602.2700

E-mail: femartin@fmrp.usp.br 